\title{
Evaluating the clinical effectiveness and safety of various HER2-targeted regimens after prior taxane/trastuzumab in patients with previously treated, unresectable, or metastatic HER2-positive breast cancer: a systematic review and network meta-analysis
}

\author{
Noman Paracha ${ }^{1}$ (D) Adriana Reyes ${ }^{1} \cdot$ Véronique Diéras $^{2} \cdot \operatorname{lan} \mathrm{Krop}^{3} \cdot$ Xavier Pivot $^{4} \cdot$ Ander Urruticoechea $^{5}$
}

Received: 29 August 2019 / Accepted: 12 February 2020 / Published online: 25 February 2020

(c) The Author(s) 2020

\begin{abstract}
Purpose In the absence of head-to-head trial data, network meta-analysis (NMA) was used to compare trastuzumab emtansine (T-DM1) with other approved treatments for previously treated patients with unresectable or metastatic HER2-positive breast cancer $(\mathrm{BC})$.

Methods Systematic reviews were conducted of published controlled trials of treatments for unresectable or metastatic HER2-positive BC with early relapse ( $\leq 6$ months) following adjuvant therapy or progression after trastuzumab (Tras) + taxane published from January 1998 to January 2018. Random-effects NMA was conducted for overall survival (OS), progression-free survival (PFS), overall response rate (ORR), and safety endpoints.

Results The NMA included regimens from seven randomized controlled trials: T-DM1 and combinations of Tras, capecitabine (Cap), lapatinib (Lap), neratinib, or pertuzumab (Per; unapproved). OS results favored T-DM1 over approved comparators: hazard ratio (HR) $(95 \%$ credible interval $[95 \%$ CrI]) vs Cap $0.68(0.39,1.10)$, LapCap 0.76 (0.51, 1.07), TrasCap $0.78(0.44,1.19)$. PFS trends favored T-DM1 over all other treatments: HR $(95 \%$ CrI $)$ vs Cap $0.38(0.19,0.74)$, LapCap 0.65 $(0.40,1.10)$, TrasCap $0.62(0.34,1.18)$; ORR with T-DM1 was more favorable than with all approved treatments. In surface under cumulative ranking curve (SUCRA) analysis T-DM1 ranked highest for all efficacy outcomes. Discontinuation due to adverse events was less likely with T-DM1 than with all comparators except neratinib. In general, gastrointestinal side effects were less likely and elevated liver transaminases and thrombocytopenia more likely with T-DM1 than with comparators. Conclusions The efficacy and tolerability profiles of T-DM1 are generally favorable compared with other treatments for unresectable or metastatic HER2-positive BC.
\end{abstract}

Keywords Trastuzumab emtansine $\cdot$ Capecitabine $\cdot$ Lapatinib $\cdot$ Neratinib $\cdot$ Pertuzumab $\cdot$ Locally advanced

Electronic supplementary material The online version of this article (https://doi.org/10.1007/s10549-020-05577-7) contains supplementary material, which is available to authorized users.

Noman Paracha

noman.paracha@roche.com

1 F. Hoffmann-La Roche AG, Grenzacherstrasse 124, 4070 Basel, Switzerland

2 Centre Eugène Marquis, Rennes, France

3 Dana-Farber Cancer Institute, Harvard Medical School, Boston, MA, USA

4 Paul Strauss Centre, Regional Institute of Cancer, Strasbourg, France

5 Onkologikoa Foundation, San Sebastián, Spain

\section{Introduction}

Human epidermal growth factor receptor 2 (HER2) is a receptor tyrosine-protein kinase, which is widely expressed and can promote tumorigenesis when expression is increased [1]; approximately $15 \%$ of breast cancer (BC) cases are HER2-positive, classified by HER2 protein overexpression or HER2 gene amplification [2]. HER2-positive BC has an aggressive clinical phenotype with, historically, a poor prognosis [3]. However, since its approval in 1998, the HER2-targeted humanized monoclonal antibody trastuzumab (Herceptin ${ }^{\circledR}$; Roche) $[4,5]$ has been associated with significant and clinically relevant improvements in diseasefree and overall survival (OS) [6]. 
Trastuzumab emtansine (T-DM1; Kadcyla ${ }^{\circledR}$; Roche) is a first-in-class antibody-drug conjugate approved for the treatment of HER2-positive unresectable locally advanced $\mathrm{BC}(\mathrm{LABC})$ or metastatic BC $(\mathrm{mBC})$ in patients previously treated with trastuzumab and a taxane, separately or in combination [7]. T-DM1 is a conjugate of DM1, a cytotoxic derivative of maytansine, and has the HER2-targeting and cytotoxicity-mediating properties of trastuzumab [8]. Conjugated DM1 is released to exert cytotoxic effects when the antibody-drug conjugate is internalized by the cell to which it binds [9].

Regulatory approval of T-DM1 for use in the $\mathrm{mBC}$ setting was based on improvements in progression-free survival (PFS) and in OS in the phase 3 EMILIA study; EMILIA was a multicenter, open-label randomized controlled trial (RCT) that evaluated the efficacy and safety of T-DM1 compared with capecitabine plus lapatinib in patients with HER2-positive $\mathrm{LABC}$ or $\mathrm{mBC}$, who were previously treated with trastuzumab and a taxane [10]. T-DM1 received US Food and Drug Administration (FDA) approval in February 2013 [11] and European Medicines Agency (EMA) marketing authorization in November 2013 [12]. A recent descriptive analysis of EMILIA, which followed up patients who crossed over from the control group to T-DM1, corroborated the original findings of improved OS with T-DM1 relative to control [13]. Additionally, based on recent data from the KATHERINE trial [14], in May 2019, T-DM1 was approved by the FDA for use in the early BC setting for patients with residual invasive disease after neoadjuvant taxane and trastuzumabbased treatment [15].

Several other therapies are available, or have been studied in patients with previously treated, unresectable, HER2positive $\mathrm{LABC}$ or $\mathrm{mBC}$ [16], including trastuzumab in combination with capecitabine [17] or vinorelbine [18], and the tyrosine kinase inhibitors lapatinib (Tyverb ${ }^{\circledR}$; Novartis-in combination with capecitabine; LapCap) $[19,20]$ and neratinib (Nerlynx ${ }^{\circledR}$; Puma Biotechnology, Inc.) [21, 22]. In the absence of direct head-to-head evidence from clinical trials, the relative efficacy of treatments can be assessed using network meta-analysis (NMA) to combine direct and indirect evidence from multiple independent trials [23]. Therefore, we conducted a systematic review (SR) and NMA to evaluate the clinical effectiveness and safety of T-DM1 versus other treatments for HER2-positive mBC. The monoclonal antibody pertuzumab (Perjeta ${ }^{\circledR}$; Roche-treatment in combination with trastuzumab and chemotherapy) [24, 25] was included in the NMA for completeness despite not being approved in this patient group. The primary endpoint analysis of the failed PHEREXA trial showed pertuzumab to increase PFS, but was not statistically significant, when added to trastuzumab plus capecitabine [26].

\section{Methods}

All SR and NMA methodology and reporting complied with Preferred Reporting Items for Systematic Reviews and Metaanalyses (PRISMA) guidelines.

\section{Systematic review}

The SR included published data between 1 January 1998 and 3 January 2018 based on searches of MEDLINE ${ }^{\circledR}$, EMBASETM $^{\mathrm{TM}}$ MEDLINE ${ }^{\circledR}$ In-Process, the Cochrane Central Register of Controlled Trials (CENTRAL), Cochrane Methods studies, the Cochrane Database of Systematic Reviews (CDSR), and the Database of Abstracts of Reviews of Effects (DARE). Database searches were complemented by manual searches of conference abstracts from the American Society of Clinical Oncology (ASCO), the European Society for Medical Oncology (ESMO), the San Antonio Breast Cancer Symposium (SABCS), and the International Society for Pharmacoeconomics and Outcomes Research (ISPOR). Studies in the SR were identified based on search strings provided in Online Resource 1. The SR searched for studies from three separate periods (1 January 1998-2 July 2013, 1 October 2012-30 June 2016, and 1 January 2016-3 January 2018), with sufficient overlap in time between the search periods to allow for indexing delays of published studies. Citations were reviewed by two independent reviewers, and discrepancies adjudicated by a third independent reviewer according to eligibility criteria presented in Online Resource 2. Eligible studies were controlled trials of pharmacological treatments for HER2-positive LABC with early relapse (within 6 months) following adjuvant therapy, or for HER2positive unresectable $\mathrm{mBC}$ in which patients had progressed after treatment with trastuzumab plus taxane. No pre-specified interventions or comparators were targeted, interventions were not required to be approved for use in this indication, and trials were included independent of randomization, phase, or blinding status. Critical appraisal of included trials was based on recommendations from the UK National Institute for Health and Care Excellence (NICE), Germany's Institute for Quality and Efficiency in Health Care (IQWIG), the Canadian Agency for Drugs and Technologies in Health (CADTH), the French National Authority for Health (HAS; randomized trials), and the Downs and Black checklist (nonrandomized trials) (Online Resource 3).

\section{Network meta-analysis}

\section{Treatment networks}

Not all studies reported all selected endpoints. Separate network plots were developed for OS, PFS, overall response 
rate (ORR), and the following safety-related endpoints (all based on treatment-related adverse events [AEs] Common Terminology Criteria for Adverse Events [CTCAE] grade 3 [severe] and above [grade 4, life-threatening or disabling; grade 5, death-related]): number of patients with AEs; serious AEs; treatment discontinuation due to AEs; and selected individual AEs, namely anemia, diarrhea, fatigue, increased alanine aminotransferase (ALT), increased aspartate aminotransferase (AST), mucosal inflammation, nausea, neutropenia, palmar-plantar erythrodysesthesia (PPE), thrombocytopenia, and vomiting.

\section{Statistical methodology}

A Bayesian NMA of PFS, OS, ORR, and safety endpoints was conducted on a log-hazard (PFS and OS) or log odds scale (ORR, safety endpoints). A Bayesian inferential framework was used, because it captures and propagates uncertainty while allowing external information to be included [27]. The NMA was conducted using the GeMTC R package in the Roche Biometrics Experimental Environment (BEE; $\mathrm{R}$ version 3.4.4) [28, 29]; both fixed-effects and randomeffects approaches were used [23]. Markov Chain Monte Carlo (MCMC) convergence was assessed by inspecting trace plots and Brooks-Gelman-Rubin statistics [30]. The detailed methodology is included in Online Resource 4 [31]. Data inputs used to calculate hazard ratios (HRs) for PFS and OS, odds ratios (ORs) for ORR and AEs, and their respective $95 \%$ credible intervals (CrIs) are included in Online Resource 5. For all endpoints, the primary analyses were based on data as reported in the relevant RCTs; for Roche-sponsored trials (EMILIA [10], GBG 26 [32], and PHEREXA [26]), unpublished data were included where relevant.

A sensitivity analysis used the treatment crossoveradjusted HR for OS, calculated using either the rank-preserving structural-failure time model (RPSFTM) [33-35] or a Cox regression model with treatment crossover as a time-dependent covariate [36]. Although there is no one ideal method for adjusting for crossover [35], RPSFTM is one of the preferred methods that preserves randomization and is least prone to selection bias [33-35]; whereas use of a Cox regression model with treatment crossover as a timedependent covariate was found to be the most robust among several approaches reported in an analysis of a phase 3 trial of LapCap versus capecitabine, and was considered to provide a more balanced result [36]. Overall rankings for each treatment were determined by estimation of Surface Under the Cumulative Ranking Curve (SUCRA; range 0-100\%) [37].

\section{Results}

\section{Study selection and heterogeneity assessment}

The results of the three rounds of SR are summarized in Fig. 1. The initial SR covered searches from 1 January 1998 to 2 July 2013. After deduplication, 3822 records were identified for screening; from which five RCTs were identified for analysis: EMILIA [10]; GBG 26 [32]; EGF100151 [38]; Martin et al. [39]; and CEREBEL [17]. The first update of the SR encompassed searches from 1 October 2012 to 30 June 2016. After deduplication, 3401 records were identified for screening, from which updates to the original five RCTs and one new RCT were identified: PHEREXA [26]. The second update to the SR covered searches from 1 January 2016 to 3 January 2018. After deduplication, 2923 records were identified for screening, from which updates and one further RCT, ELTOP [40], were identified.

Overall, seven RCTs met the criteria for inclusion in the NMA: EMILIA [10]; GBG 26 [32]; EGF100151 [38]; a phase 2 trial of neratinib versus lapatinib plus capecitabine (Martin et al. 2013) [39]; PHEREXA [26]; ELTOP [40]; and a prior trastuzumab treatment subgroup in CEREBEL [17]although CEREBEL did not meet the inclusion criteria, it included a subgroup defined as "patients who received prior trastuzumab either in adjuvant or metastatic setting" and was thus included in the NMA. The phase 3 TH3RESA study [41] that evaluated T-DM1 was excluded from the NMA because of limitations that its inclusion would impose i.e., only patients on certain treatment regimens in the comparator arm "Physician's choice" were relevant to the NMA, and disaggregating data on particular treatments from this arm would have broken randomization. Thus, selecting a particular treatment from "Physician's choice" would introduce bias and break the fundamental principle of NMA which relies on randomized evidence.

Eligibility criteria for inclusion in the NMA are summarized in Online Resource 2, and trial information and patient characteristics at baseline in the trials, which were included in the analysis, are summarized in Table 1. Although there were differences in population size and treatment line among studies, heterogeneity assessment indicated that all trials were comparable in terms of randomization, allocation concealment, demographic and baseline characteristics, outcome selection and reporting, patient withdrawal from the studies, and statistical analyses undertaken (Online Resource 3). In total, there were five phase 3 studies and two phase 2 studies. All were open-label, but EMILIA and EGF100151 used independent review committees to assess outcomes and, therefore, the outcome assessors were blinded to study treatment. Patients from all studies had been treated previously with trastuzumab; however, only results from a "prior 
Fig. 1 PRISMA diagram of included and excluded studies ( ${ }^{\mathrm{a}}$ Respectively, the record numbers shown are those from the initial SR covering 1 January 1998 to 2 July 2013, from the first update of the SR, covering 1 October 2012 to 30 June 2016, and from the second update of the SR, covering 1 January 2016 to 3 January 2018 ${ }^{\mathrm{b}}$ For each clinical study only the primary references, and not conference abstracts, are cited in the text). A PRISMA checklist is provided in Online Resource 11. PRISMA Preferred Reporting Items for Systematic Reviews and Meta-analyses, $S R$ systematic review

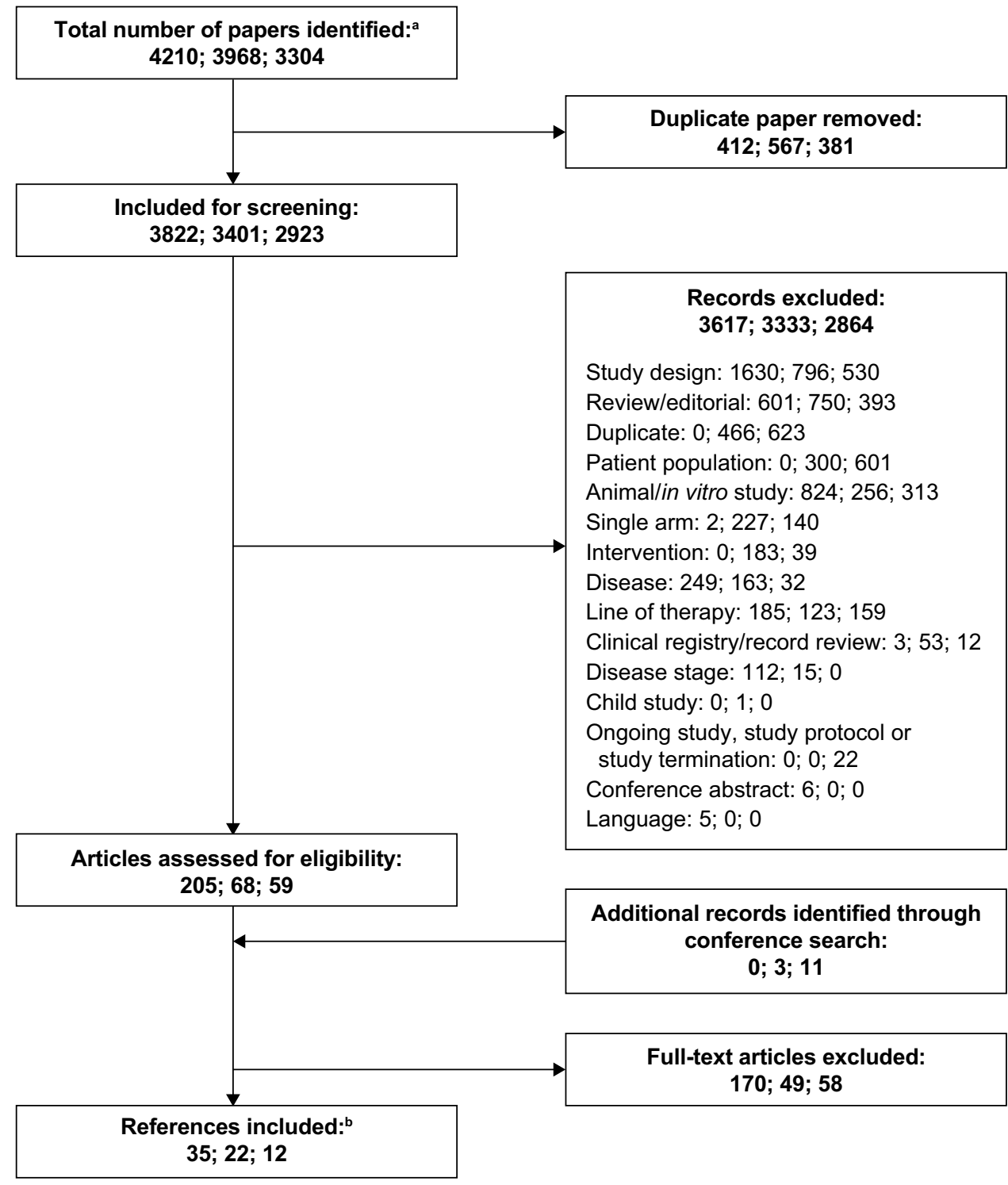

trastuzumab treatment" subgroup were included from the CEREBEL study. Results of critical appraisal of trials are presented in Online Resource 3.

\section{Treatment networks}

Seven studies reported data for OS, for OS adjusted for treatment crossover, and for PFS (Fig. 2a); six studies for ORR as data were not reported in CEREBEL (Fig. 2b). Various treatment network plots were generated for the safety endpoints (Online Resource 6). Six studies were linked to network plots of treatment discontinuation (due to AEs), diarrhea, neutropenia, and ALT. Five studies were linked to plots for fatigue, nausea, and vomiting, four studies were included for AEs (grade 3 and above) and AST, and two studies were linked for serious AEs (Online Resource 6).

\section{Model selection}

The Bayesian random-effects model was the base-case analysis, and was preferred over the fixed-effects model for all endpoints to account for heterogeneity among the included studies. Between-study variance cannot be estimated owing to the small number of available studies and assuming homogeneity was considered to be implausible. Hence, informative priors based on the best empirical evidence were used instead [42]. Convergence statistics for the random-effects model are shown in Online Resource 8, and convergence plots are presented in Online Resource 10. For completeness, results obtained with the fixed-effects model are shown in Online Resource 9. 
Table 1 Trial methodologies and baseline characteristics

\begin{tabular}{|c|c|c|c|c|c|c|c|}
\hline & $\begin{array}{l}\text { EMILIA [10] } \\
\text { NCT00829166 }\end{array}$ & $\begin{array}{l}\text { GBG } 26 \text { [32] } \\
\text { NCT00148876 }\end{array}$ & $\begin{array}{l}\text { EGF100151 [36, } \\
\text { 38] NCT00078572 }\end{array}$ & $\begin{array}{l}\text { Martin et al., } 2013 \\
\text { [39] } \\
\text { NCT00777101 }\end{array}$ & $\begin{array}{l}\text { CEREBEL trial } \\
\text { subgroup }^{\mathrm{a}}[17] \\
\text { NCT00820222 }^{2}\end{array}$ & $\begin{array}{l}\text { PHEREXA [26] } \\
\text { NCT01026142 }\end{array}$ & ELTOP [40] \\
\hline Intervention & $\begin{array}{l}\text { T-DM1 } \\
(N=495)\end{array}$ & $\begin{array}{l}\text { Cap + trastu- } \\
\text { zumab } \\
(N=78)\end{array}$ & $\begin{array}{l}\text { Cap+lap } \\
(N=198)\end{array}$ & $\begin{array}{l}\text { Neratinib } \\
(N=117)\end{array}$ & $\begin{array}{l}\text { Cap+lap } \\
(N=271)\end{array}$ & $\begin{array}{l}\text { Pertuzumab + tras- } \\
\text { tuzumab + cap } \\
(N=228)\end{array}$ & $\begin{array}{l}\text { Cap + trastu- } \\
\text { zumab } \\
(N=43)\end{array}$ \\
\hline Comparator & $\begin{array}{l}\text { Cap+lap } \\
(N=496)\end{array}$ & $\begin{array}{l}\text { Cap } \\
(N=78)\end{array}$ & $\begin{array}{l}\text { Cap } \\
(N=201)\end{array}$ & $\begin{array}{l}\text { Cap+lap } \\
(N=116)\end{array}$ & $\begin{array}{l}\text { Cap + trastuzumab } \\
(N=269)\end{array}$ & $\begin{array}{c}\text { Cap + trastuzumab } \\
(N=224)\end{array}$ & $\begin{array}{l}\text { Cap +lap } \\
(N=43)\end{array}$ \\
\hline $\begin{array}{l}\text { Crossover permit- } \\
\text { ted, } N(\%)\end{array}$ & Yes $(27 \%)[13]$ & No & $\begin{array}{l}\text { Yes }(18 \%)^{\mathrm{j}} \\
{[36]}\end{array}$ & No & No & No & No \\
\hline \multicolumn{8}{|c|}{ Present line of treatment, $N(\%)$} \\
\hline $1 \mathrm{~L}$ & 0 & 0 & NR & 0 & $238(44)$ & 0 & $5(6)$ \\
\hline $1 \mathrm{~L}-\mathrm{R}$ & $118(12)$ & 0 & NR & 0 & NR & 0 & NR \\
\hline $2 \mathrm{~L}$ & $361(36)$ & $156(100)$ & $393(98)$ & $32(14)$ & $302(56)^{\mathrm{b}}$ & $449(100)^{\mathrm{c}}$ & $61(71)$ \\
\hline $3 \mathrm{~L}$ or later & $512(52)$ & NR & NR & $200(86)^{b}$ & NR & 0 & $20(23)$ \\
\hline $\begin{array}{l}\text { Age, median years } \\
\text { (range) }\end{array}$ & $\begin{array}{l}\text { I: } 53(25-84) \\
\text { C: } 53(24-83)\end{array}$ & $\begin{array}{l}\text { I: } 53(28-78) \\
\text { C: } 59(33-82)\end{array}$ & $\begin{array}{l}\text { I: } 54(26-80) \\
\text { C: } 51(28-83)\end{array}$ & $\begin{array}{l}\text { I: } 52(28-79) \\
\text { C: } 56(30-79)\end{array}$ & $\begin{array}{l}\text { I: } 53(27-83) \\
\text { C: } 56(31-79)\end{array}$ & $\begin{array}{l}\text { I: } 54 \text { (NR) } \\
\text { C: } 55 \text { (NR) }\end{array}$ & $\begin{array}{l}\text { I: } 57(34-81) \\
\text { C: } 59(37-78)\end{array}$ \\
\hline $\begin{array}{l}\text { ECOG perfor- } \\
\text { mance status }=1 \text {, } \\
N(\%)\end{array}$ & $\begin{array}{l}\text { I: } 194(39)^{\mathrm{d}} \\
\text { C: } 176(35)^{\mathrm{d}}\end{array}$ & NR & $\begin{array}{l}\text { I: } 76(38) \\
\text { C: } 83(41)\end{array}$ & $\begin{array}{l}\text { I: } 43(37) \\
\text { C: } 39(34)\end{array}$ & $\begin{array}{l}\text { I: } 260(96)^{\mathrm{e}} \\
\text { C: } 261(98)^{\mathrm{e}}\end{array}$ & $\begin{array}{l}\text { I: } 68(30) \\
\text { C: } 73(33)\end{array}$ & $\begin{array}{l}\text { I: } 18(42) \\
\text { C: } 12(28)\end{array}$ \\
\hline $\begin{array}{c}\mathrm{ER}+\text { and/or } \mathrm{PR}+ \\
\text { tumors, } N(\%)\end{array}$ & $\begin{array}{l}\text { I: } 282(57)^{\mathrm{f}} \\
\text { C: } 263(53)^{\mathrm{f}}\end{array}$ & $\begin{array}{l}\text { I: } 41(56)^{\mathrm{g}} \\
\text { C: } 43(62)^{\mathrm{g}}\end{array}$ & $\begin{array}{l}\text { I: } 96(48) \\
\text { C: } 93(46)\end{array}$ & $\begin{array}{l}\text { I: } 52 \text { (44) ER+; } \\
31 \text { (27) PR+ } \\
\text { C: } 46 \text { (40) ER+; } \\
32 \text { (28) PR+ }\end{array}$ & $\begin{array}{l}\text { I: } 133 \text { (49) ER+; } \\
98 \text { (36) PR+ } \\
\text { C: } 122(45) \text { ER+; } \\
80 \text { (30) PR+ }\end{array}$ & $\begin{array}{l}\text { I: } 126(55) \\
\text { C: } 123(55)\end{array}$ & $\begin{array}{l}\text { I: } 27(63) \\
\text { C: } 27(63)\end{array}$ \\
\hline $\begin{array}{l}\text { Time since } \\
\text { initial diagnosis, } \\
\text { median years } \\
\text { (range) }\end{array}$ & $\begin{array}{l}\text { C: } 3.1(0.1-29.8) \\
\text { I: } 3.3(0.2-31.6)\end{array}$ & NR & $\begin{array}{l}\text { I: } 3.8(0-21)^{\mathrm{h}} \\
\text { C: } 4.1(0-19)\end{array}$ & NR & $\begin{array}{l}\text { I: } 2.6(0-18) \\
\text { C: } 3.0(0-25)\end{array}$ & NR & NR \\
\hline $\begin{array}{l}\text { Time since first } \\
\text { metastases, } \\
\text { median years } \\
\text { (range) }\end{array}$ & $\begin{array}{l}\text { C: } 1.5(0.04-15.1) \\
\text { I: } 1.3(0.03-24.3)\end{array}$ & NR & $\begin{array}{l}\text { I: } 1.70(0-9) \\
\text { C: } 1.60(0-8)\end{array}$ & NR & NR & NR & NR \\
\hline $\begin{array}{l}\text { Advanced or meta- } \\
\text { static sites in the } \\
\text { brain, } N(\%)\end{array}$ & $\begin{array}{l}\text { C: } 45(9) \\
\text { I: } 50(10)\end{array}$ & $\begin{array}{l}\text { I: } 1(1) \\
\text { C: } 2(3)\end{array}$ & NR & NR & $\begin{array}{l}\text { I: } 0 \\
\text { C: } 0\end{array}$ & $\begin{array}{l}\text { I: } 25(11) \\
\text { C: } 28(13)\end{array}$ & $\begin{array}{l}\text { I: } 6(14) \\
\text { C: } 7(16)\end{array}$ \\
\hline $\begin{array}{l}\text { Visceral disease, } \\
N(\%)\end{array}$ & $\begin{array}{l}\text { I: } 334(67) \\
\text { C: } 335(68)\end{array}$ & NR & $\begin{array}{l}\text { I: } 153(77) \\
\text { C: } 158(79)\end{array}$ & NR & $\begin{array}{l}\text { I: } 173(64) \\
\text { C: } 164(61)\end{array}$ & $\begin{array}{l}\text { I: } 148(65)^{\mathrm{i}} \\
\text { C: } 146(65)^{\mathrm{i}}\end{array}$ & NR \\
\hline
\end{tabular}

$1 L$ first line, $1 L-R$ first-line relapse, $2 L$ second line, $3 L$ third line, $C$ comparator, Cap capecitabine, $C N S$ central nervous system, $E C O G$ Eastern Cooperative Oncology Group, $E R$ estrogen receptor, GBG German Breast Group, I intervention, Lap lapatinib, $N R$ not reported, $P R$ progesterone receptor, $T$-DMI trastuzumab emtansine

${ }^{a}$ The information shown is for the total intent-to-treat population for CEREBEL; however, analysis of progression-free and overall survival is based on only the subgroup of patients who received prior trastuzumab in the adjuvant or metastatic setting $(N=167$ for cap + lap and $N=159$ for cap + trastuzumab)

${ }^{\mathrm{b}}$ Patients with two or three or more prior anti-cancer regimens

${ }^{\mathrm{c}} 100 \%$ of patients received trastuzumab in the $1 \mathrm{~L}$ metastatic breast cancer setting; information on prior trastuzumab setting missing in three patients

${ }^{\mathrm{d}}$ ECOG performance status not available for eight and two patients in cap + lap and T-DM1 groups, respectively

${ }^{\mathrm{e}} \mathrm{ECOG}$ performance status of 0 or 1

${ }^{\mathrm{f}}$ Hormone receptor status unknown for nine and 11 patients in cap + lap and T-DM1 groups, respectively

${ }^{\mathrm{g}}$ Hormone receptor status was not reported for five and seven patients in the cap + trastuzumab and cap groups, respectively

${ }^{\text {h}}$ Value based on $N=207 ; 198$ patients were randomized to cap + lap and nine additional patients were assigned the treatment later on during the trial

${ }^{i}$ Percentage based on a total $N$ of 223 and 226 in the cap + trastuzumab and pertuzumab + trastuzumab + cap groups, respectively

${ }^{\mathrm{j}}$ Percentage based on a total $N$ of 201 and a total of 36 patients that crossed over to the combination arm 
Fig. 2 Treatment network plots for a OS, adjusted OS, PFS, and b ORR. Cap capecitabine, Lap lapatinib, $O R R$ overall response rate, $O S$ overall survival, $P e r$ pertuzumab, $P F S$ progression-free survival, $T-D M 1$ trastuzumab emtansine, Tras trastuzumab
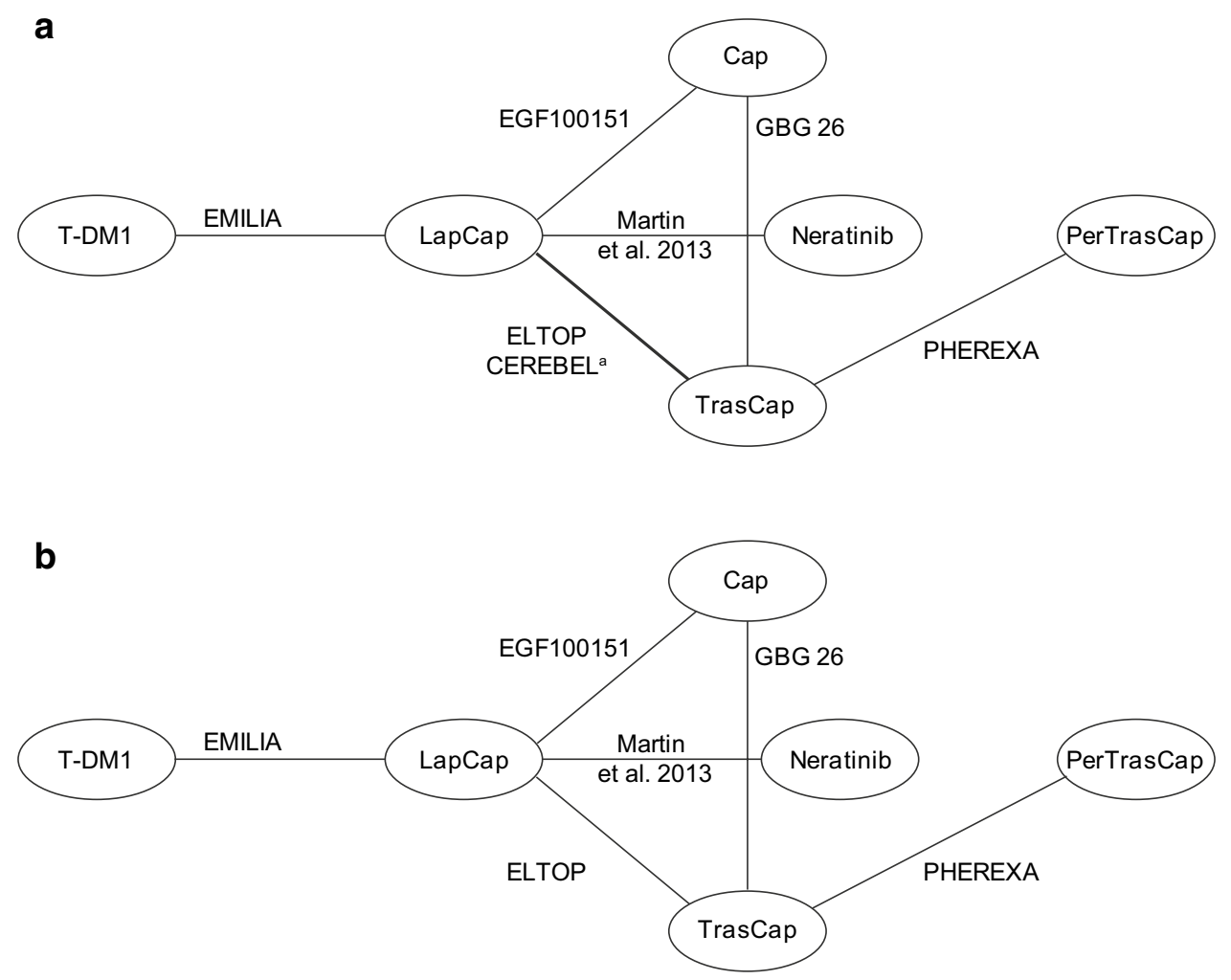

\section{Overall survival}

The HR data for cross-comparison of treatments are summarized for OS (the primary analysis) and for OS adjusted for treatment crossover (sensitivity analysis) in Table 2 and Fig. 3.

\section{Primary analysis}

T-DM1 was associated with a trend towards greater OS benefit than all other approved treatments, although the wide CrIs reflect uncertainty around the comparisons. Greater OS benefit with T-DM1 was also demonstrated by the SUCRA ranking (first), compared with other approved treatments: (1) T-DM1, (2) pertuzumab plus trastuzumab plus capecitabine (unapproved combination), (3) trastuzumab plus capecitabine, (4) lapatinib plus capecitabine, (5) capecitabine, and (6) neratinib.

\section{Sensitivity analysis}

In the sensitivity analysis, adjusted estimates of OS were available for both EMILIA and EGF100151 (Online Resource 5) [10, 36]. For EMILIA, the treatment crossoveradjusted HR for OS was 0.69 (95\% confidence interval [CI] 0.59, 0.82) using RPSFTM. In EGF100151, the adjusted HR for OS in which treatment crossover was used as a time-dependent covariate was 0.80 (95\% CI 0.64, 0.99). Intention-to-treat estimates of OS were used for the other five studies, as in the primary analysis [17, 32, 38-40]. Sensitivity analysis results were generally similar to the basecase analysis, with a numerically greater OS benefit for T-DM1 than for the other treatments (Table 2 and Fig. 3).

\section{Progression-free survival}

Cross-comparison, between-treatment HRs for PFS are also summarized in Table 2 and Fig. 3. The analysis indicated that the likelihood of PFS benefit was greater with T-DM1 than with any of the other comparator treatments. The SUCRA ranking was also greater for T-DM1 (first) than for the other approved treatments: (1) T-DM1, (2) pertuzumab plus trastuzumab plus capecitabine, (3) lapatinib plus capecitabine, (4) trastuzumab plus capecitabine, (5) neratinib, and (6) capecitabine.

\section{Overall response rates}

Comparisons of ORR with T-DM1 and with other treatments showed that T-DM1 was associated with a more favorable ORR than all comparator treatments, and was more efficacious than capecitabine, lapatinib plus capecitabine, and neratinib (Fig. 4). Consistent with this finding, the SUCRA ranking was greatest for T-DM1 compared with the other 
Table 2 Cross tabulation of treatment HR (95\% CrI) for OS, OSX, and PFS

\begin{tabular}{|c|c|c|c|c|c|c|}
\hline Drug & T-DM1 & Neratinib & Cap & LapCap & TrasCap & PerTrasCap \\
\hline \multirow[t]{3}{*}{ T-DM1 } & & $\begin{array}{l}\text { OS: } 0.60 \\
(0.32,1.14)\end{array}$ & $\begin{array}{l}\text { OS: } 0.68 \\
(0.39,1.10)\end{array}$ & $\begin{array}{l}\text { OS: } 0.76 \\
(0.51,1.07)\end{array}$ & $\begin{array}{l}\text { OS: } 0.78 \\
(0.44,1.19)\end{array}$ & $\begin{array}{l}\text { OS: } 1.03 \\
(0.51,1.82)\end{array}$ \\
\hline & & $\begin{array}{l}\text { OSX: } 0.56 \\
(0.28,1.10)\end{array}$ & $\begin{array}{l}\text { OSX: } 0.59 \\
(0.33,1.00)\end{array}$ & $\begin{array}{l}\text { OSX: } 0.69 \\
(0.46,1.08)\end{array}$ & $\begin{array}{l}\text { OSX: } 0.70 \\
(0.38,1.16)\end{array}$ & $\begin{array}{l}\text { OSX: } 0.93 \\
(0.42,1.73)\end{array}$ \\
\hline & & $\begin{array}{l}\text { PFS: } 0.55 \\
(0.28,1.24)\end{array}$ & $\begin{array}{l}\text { PFS: } 0.38 \\
(0.19,0.74)\end{array}$ & $\begin{array}{l}\text { PFS: } 0.65 \\
(0.40,1.10)\end{array}$ & $\begin{array}{l}\text { PFS: } 0.62 \\
(0.34,1.18)\end{array}$ & $\begin{array}{l}\text { PFS: } 0.74 \\
(0.32,1.76)\end{array}$ \\
\hline \multirow[t]{3}{*}{ Neratinib } & $\begin{array}{l}\text { OS: } 1.65 \\
(0.88,3.11)\end{array}$ & & $\begin{array}{l}\text { OS: } 1.13 \\
(0.58,2.09)\end{array}$ & $\begin{array}{l}\text { OS: } 1.25 \\
(0.76,2.13)\end{array}$ & $\begin{array}{l}\text { OS: } 1.30 \\
(0.67,2.35)\end{array}$ & $\begin{array}{l}\text { OS: } 1.70 \\
(0.79,3.54)\end{array}$ \\
\hline & $\begin{array}{l}\text { OSX: } 1.80 \\
(0.91,3.57)\end{array}$ & & $\begin{array}{l}\text { OSX: } 1.05 \\
(0.52,2.01)\end{array}$ & $\begin{array}{l}\text { OSX: } 1.25 \\
(0.72,2.10)\end{array}$ & $\begin{array}{l}\text { OSX: } 1.26 \\
(0.65,2.24)\end{array}$ & $\begin{array}{l}\text { OSX: } 1.66 \\
(0.76,3.29)\end{array}$ \\
\hline & $\begin{array}{l}\text { PFS: } 1.82 \\
(0.81,3.58)\end{array}$ & & $\begin{array}{l}\text { PFS: } 0.69 \\
(0.33,1.34)\end{array}$ & $\begin{array}{l}\text { PFS: } 1.18 \\
(0.65,2.01)\end{array}$ & $\begin{array}{l}\text { PFS: } 1.12 \\
(0.55,2.10)\end{array}$ & $\begin{array}{l}\text { PFS: } 1.33 \\
(0.56,2.92)\end{array}$ \\
\hline \multirow[t]{3}{*}{ Cap } & $\begin{array}{l}\text { OS: } 1.47 \\
(0.91,2.54)\end{array}$ & $\begin{array}{l}\text { OS: } 0.88 \\
(0.48,1.72)\end{array}$ & & $\begin{array}{l}\text { OS: } 1.11 \\
(0.79,1.63)\end{array}$ & $\begin{array}{l}\text { OS: } 1.15 \\
(0.76,1.61)\end{array}$ & $\begin{array}{l}\text { OS: } 1.51 \\
(0.82,2.59)\end{array}$ \\
\hline & $\begin{array}{l}\text { OSX: } 1.70 \\
(1.00,3.04)\end{array}$ & $\begin{array}{l}\text { OSX: } 0.95 \\
(0.50,1.93)\end{array}$ & & $\begin{array}{l}\text { OSX: } 1.18 \\
(0.85,1.69)\end{array}$ & $\begin{array}{l}\text { OSX: } 1.19 \\
(0.79,1.68)\end{array}$ & $\begin{array}{l}\text { OSX: } 1.57 \\
(0.84,2.66)\end{array}$ \\
\hline & $\begin{array}{l}\text { PFS: } 2.62 \\
(1.35,5.19)\end{array}$ & $\begin{array}{l}\text { PFS: } 1.45 \\
(0.75,2.99)\end{array}$ & & $\begin{array}{l}\text { PFS: } 1.71 \\
(1.11,2.72)\end{array}$ & $\begin{array}{l}\text { PFS: } 1.62 \\
(1.02,2.56)\end{array}$ & $\begin{array}{l}\text { PFS: } 1.94 \\
(0.96,3.81)\end{array}$ \\
\hline \multirow[t]{3}{*}{ LapCap } & $\begin{array}{l}\text { OS: } 1.32 \\
(0.93,1.98)\end{array}$ & $\begin{array}{l}\text { OS: } 0.80 \\
(0.47,1.32)\end{array}$ & $\begin{array}{l}\text { OS: } 0.90 \\
(0.61,1.26)\end{array}$ & & $\begin{array}{l}\text { OS: } 1.04 \\
(0.73,1.37)\end{array}$ & $\begin{array}{l}\text { OS: } 1.36 \\
(0.77,2.19)\end{array}$ \\
\hline & $\begin{array}{l}\text { OSX: } 1.45 \\
(0.93,2.19)\end{array}$ & $\begin{array}{l}\text { OSX: } 0.80 \\
(0.48,1.39)\end{array}$ & $\begin{array}{l}\text { OSX: } 0.85 \\
(0.59,1.18)\end{array}$ & & $\begin{array}{l}\text { OSX: } 1.01 \\
(0.68,1.35)\end{array}$ & $\begin{array}{l}\text { OSX: } 1.34 \\
(0.74,2.18)\end{array}$ \\
\hline & $\begin{array}{l}\text { PFS: } 1.54 \\
(0.91,2.52)\end{array}$ & $\begin{array}{l}\text { PFS: } 0.85 \\
(0.50,1.54)\end{array}$ & $\begin{array}{l}\text { PFS: } 0.59 \\
(0.37,0.90)\end{array}$ & & $\begin{array}{l}\text { PFS: } 0.95 \\
(0.66,1.30)\end{array}$ & $\begin{array}{l}\text { PFS: } 1.13 \\
(0.57,2.17)\end{array}$ \\
\hline \multirow[t]{3}{*}{ TrasCap } & $\begin{array}{l}\text { OS: } 1.28 \\
(0.84,2.27)\end{array}$ & $\begin{array}{l}\text { OS: } 0.77 \\
(0.42,1.49)\end{array}$ & $\begin{array}{l}\text { OS: } 0.87 \\
(0.62,1.32)\end{array}$ & $\begin{array}{l}\text { OS: } 0.96 \\
(0.73,1.37)\end{array}$ & & $\begin{array}{l}\text { OS: } 1.31 \\
(0.85,2.01)\end{array}$ \\
\hline & $\begin{array}{l}\text { OSX: } 1.42 \\
(0.86,2.60)\end{array}$ & $\begin{array}{l}\text { OSX: } 0.80 \\
(0.45,1.55)\end{array}$ & $\begin{array}{l}\text { OSX: } 0.84 \\
(0.59,1.27)\end{array}$ & $\begin{array}{l}\text { OSX: } 0.99 \\
(0.74,1.47)\end{array}$ & & $\begin{array}{l}\text { OSX: } 1.32 \\
(0.85,2.02)\end{array}$ \\
\hline & $\begin{array}{l}\text { PFS: } 1.62 \\
(0.85,2.97)\end{array}$ & $\begin{array}{l}\text { PFS: } 0.89 \\
(0.48,1.83)\end{array}$ & $\begin{array}{l}\text { PFS: } 0.62 \\
(0.39,0.98)\end{array}$ & $\begin{array}{l}\text { PFS: } 1.05 \\
(0.77,1.53)\end{array}$ & & $\begin{array}{l}\text { PFS: } 1.20 \\
(0.71,1.94)\end{array}$ \\
\hline \multirow[t]{3}{*}{ PerTrasCap } & $\begin{array}{l}\text { OS: } 0.97 \\
(0.55,1.96)\end{array}$ & $\begin{array}{l}\text { OS: } 0.59 \\
(0.28,1.26)\end{array}$ & $\begin{array}{l}\text { OS: } 0.66 \\
(0.39,1.22)\end{array}$ & $\begin{array}{l}\text { OS: } 0.74 \\
(0.46,1.30)\end{array}$ & $\begin{array}{l}\text { OS: } 0.76 \\
(0.50,1.17)\end{array}$ & \\
\hline & $\begin{array}{l}\text { OSX: } 1.07 \\
(0.58,2.37)\end{array}$ & $\begin{array}{l}\text { OSX: } 0.60 \\
(0.30,1.32)\end{array}$ & $\begin{array}{l}\text { OSX: } 0.64 \\
(0.38,1.19)\end{array}$ & $\begin{array}{l}\text { OSX: } 0.75 \\
(0.46,1.36)\end{array}$ & $\begin{array}{l}\text { OSX: } 0.76 \\
(0.50,1.18)\end{array}$ & \\
\hline & $\begin{array}{l}\text { PFS: } 1.34 \\
(0.57,3.09)\end{array}$ & $\begin{array}{l}\text { PFS: } 0.75 \\
(0.34,1.78)\end{array}$ & $\begin{array}{l}\text { PFS: } 0.52 \\
(0.26,1.04)\end{array}$ & $\begin{array}{l}\text { PFS: } 0.88 \\
(0.46,1.77)\end{array}$ & $\begin{array}{l}\text { PFS: } 0.83 \\
(0.52,1.41)\end{array}$ & \\
\hline
\end{tabular}

$\mathrm{HR}<1$ indicates a better outcome with the drug in column 1 than with the comparator drug (columns 2-7)

Cap capecitabine, CrI credible interval, HR hazard ratio, Lap lapatinib, OS overall survival, OSX OS adjusted for crossover, Per pertuzumab, PFS progression-free survival, T-DMI trastuzumab emtansine, Tras trastuzumab

approved treatments: (1) T-DM1, (2) pertuzumab plus trastuzumab plus capecitabine, (3) trastuzumab plus capecitabine, (4) lapatinib plus capecitabine, (5) neratinib, and (6) capecitabine.

\section{Adverse events (grade 3 and above)}

The ORs for the likelihood of various AEs occurring with T-DM1 compared with the different comparator treatments are summarized in Fig. 5. Treatment discontinuation due to an AE of grade 3 and above was less likely with T-DM1 than with other treatments that could be compared (there was no link between neratinib and T-DM1 in the network, and these therapies could not be compared), and discontinuation due to any AE was less likely with T-DM1 than with all other treatments except for neratinib. The SUCRA rankings for discontinuation due to an AE of grade 3 and above for approved treatments were: (1) T-DM1, (2) pertuzumab plus trastuzumab plus capecitabine, (3) trastuzumab plus capecitabine, (4) lapatinib plus capecitabine, and (5) capecitabine. The likelihood of serious AEs was lower with T-DM1 than with neratinib, or lapatinib plus capecitabine; no comparison with other treatments was possible (Fig. 5a).

The ORs indicated a substantially lower risk of diarrhea associated with T-DM1 than with other treatments, and this difference was reflected by the SUCRA rankings: (1) 
HR (95\% Crl)

\begin{tabular}{ll} 
OS (unadjusted) & \\
PerTrasCap & $1.03(0.51,1.82)$ \\
TrasCap & $0.78(0.44,1.19)$ \\
LapCap & $0.76(0.51,1.07)$ \\
Cap & $0.68(0.39,1.10)$ \\
Neratinib & $0.60(0.32,1.11)$ \\
\hline OS (adjusted) & \\
PerTrasCap & $0.93(0.42,1.73)$ \\
LapCap & $0.69(0.46,1.08)$ \\
TrasCap & $0.70(0.38,1.16)$ \\
Neratinib & $0.56(0.28,1.10)$ \\
Cap & $0.59(0.33,1.00)$ \\
PFS & \\
PerTrasCap & $0.74(0.32,1.76)$ \\
LapCap & $0.65(0.40,1.10)$ \\
TrasCap & $0.62(0.34,1.18)$ \\
Neratinib & $0.55(0.28,1.24)$ \\
Cap & $0.38(0.19,0.74)$
\end{tabular}

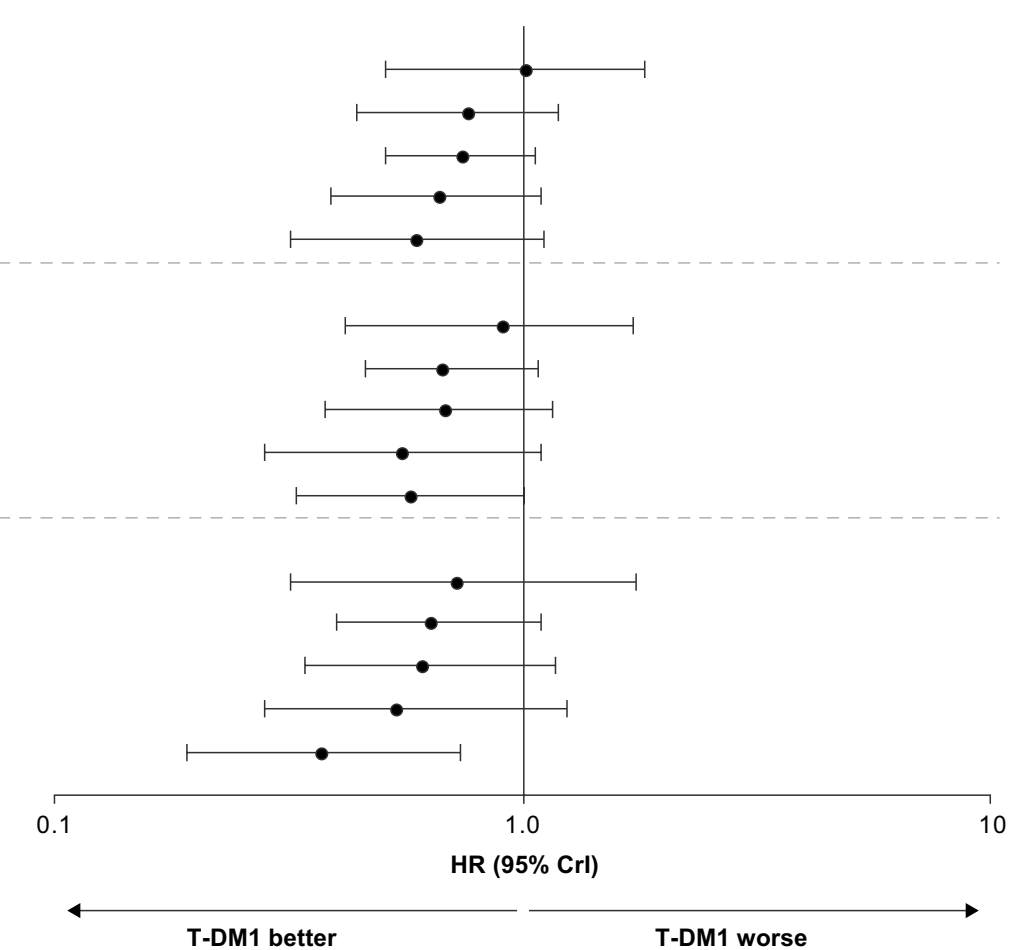

Fig. 3 Comparative effectiveness of T-DM1 on OS, adjusted OS, and PFS. Comparators are shown in order of SUCRA ranking, with treatments ranking highest after T-DM1 at the top of the plot. Cap capecitabine, $C r I$ credible interval, $H R$ hazard ratio, Lap lapatinib,

OS overall survival, $\mathrm{Per}$ pertuzumab, PFS progression-free survival, SUCRA surface under cumulative ranking curve, T-DM1 trastuzumab emtansine, Tras trastuzumab

\section{OR (95\% Crl)}

$\begin{array}{ll}\text { ORR vs T-DM1 } & \\ \text { PerTrasCap } & 1.10(0.39,3.19) \\ \text { TrasCap } & 1.49(0.67,3.61) \\ \text { LapCap } & 1.72(1.02,2.88) \\ \text { Neratinib } & 3.54(1.63,7.50) \\ \text { Cap } & 2.88(1.19,7.37)\end{array}$

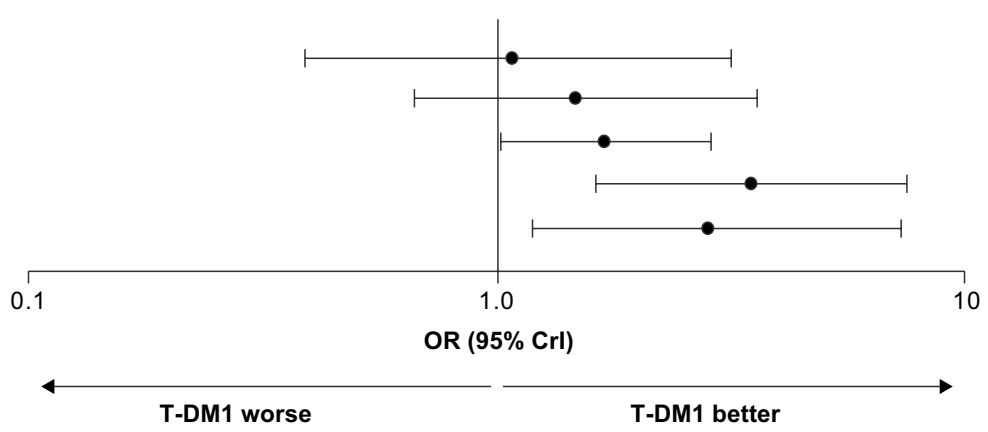

Fig. 4 Comparative effectiveness of T-DM1 on ORR. Comparators are shown in order of SUCRA ranking, with treatments ranking highest after T-DM1 at the top of the plot. Cap capecitabine, $\mathrm{CrI}$ credible

interval, Lap lapatinib, $O R$ odds ratio, $O R R$ overall response rate, $P e r$ pertuzumab, SUCRA surface under cumulative ranking curve, T-DM1 trastuzumab emtansine, Tras trastuzumab

T-DM1, (2) capecitabine, (3) trastuzumab plus capecitabine, (4) lapatinib plus capecitabine, (5) pertuzumab plus trastuzumab plus capecitabine, and (6) neratinib. Most ORs for fatigue, nausea, vomiting, and neutropenia favored T-DM1 over other treatments; however, there was higher uncertainty for lower risk of vomiting with T-DM1 than with neratinib, or lapatinib plus capecitabine (Fig. 5b).

ORs indicated that increased AST was more likely with T-DM1 than with other treatments, and was least likely with capecitabine. The SUCRA rankings were: (1) capecitabine, (2) lapatinib plus capecitabine, (3) neratinib, (4) trastuzumab plus capecitabine, and (5) T-DM1. The risk of increased ALT with T-DM1 was higher than with trastuzumab plus capecitabine, lapatinib plus capecitabine, or capecitabine. 
a

\begin{tabular}{lr} 
& \multicolumn{1}{c}{ OR $(95 \%$ Crl) } \\
Discontinuation, grade 3+ AE & $0.83(0.08,8.26)$ \\
PerTrasCap & $0.64(0.07,5.26)$ \\
TrasCap & $0.62(0.21,1.89)$ \\
LapCap & $0.58(0.11,2.91)$ \\
Cap & \\
SAE & $0.91(0.28,3.07)$ \\
Lap Cap & $0.68(0.11,3.93)$ \\
Neratinib & \\
Discontinuation, any AE & $2.75(0.44,17.84)$ \\
$\quad$ Neratinib & $0.81(0.23,2.68)$ \\
LapCap & $0.60(0.11,2.65)$ \\
Cap & $0.48(0.07,2.16)$ \\
TrasCap & $0.41(0.05,2.70)$ \\
PerTrasCap &
\end{tabular}

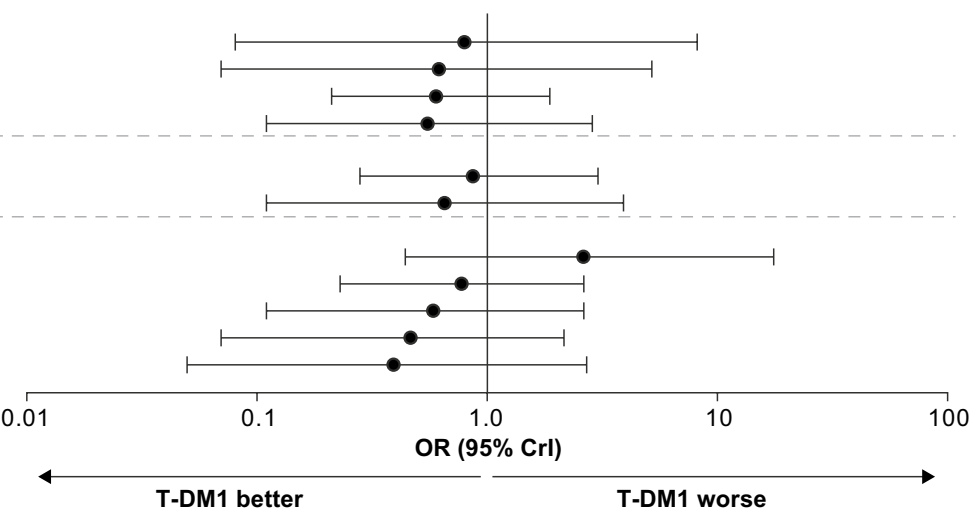

b

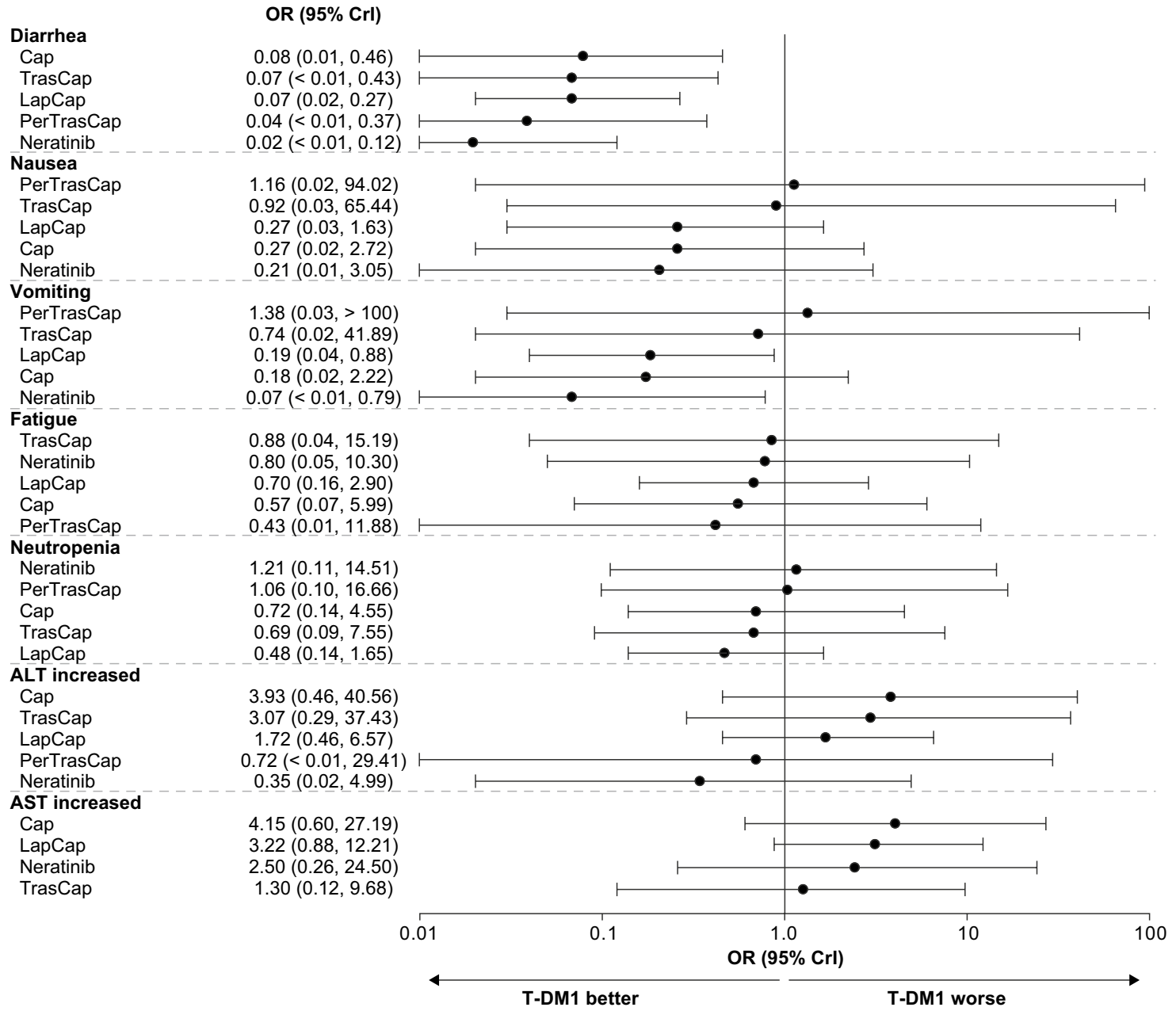

Fig. 5 Comparative effectiveness of T-DM1 on AEs: a SAEs and AEs leading to discontinuation; b AEs by preferred term. Comparators are shown in order of SUCRA ranking, with treatments ranking highest at the top of the plot. T-DM1 ranks: discontinuation (grade $3+$ ), SAEs, diarrhea, nausea, fatigue, first; vomiting, second; neutropenia, third; increased ALT, fourth; increased AST, fifth. AE adverse event, $A L T$ alanine aminotransferase, $A S T$ aspartate aminotransferase, Cap capecitabine, $\mathrm{CrI}$ credible interval, Lap lapatinib, $O R$ odds ratio, Per pertuzumab, SAE serious adverse event, SUCRA surface under cumulative ranking curve, $T$-DM1 trastuzumab emtansine, Tras trastuzumab 
Effect size could not be quantified for mucosal inflammation. T-DM1 was consistently better than the comparators, but no mucosal inflammation events were reported in one of the cohorts in study EGF100151; removal of this study disconnected the network, preventing further analysis. Similarly, the absence of events in one arm of study GBG 26 prevented further analysis of thrombocytopenia; T-DM1 was consistently worse than the comparators but this could not be quantified, even when GBG 26 was removed from the network. T-DM1 was also consistently worse than comparators in terms of anemia events. The absence of such AEs in one arm of GBG 26 afforded the possibility to re-analyze with that study excluded; OR estimates for T-DM1 are shown in Online Resource 7. Finally, no effect size could be estimated for PPE, owing to PPE being a rare event in EMILIA, thereby preventing estimation of an OR.

\section{Discussion}

This SR and NMA of RCTs undertaken in previously treated patients with unresectable, HER2-positive LABC or mBC represents the first synthesis of clinical effectiveness and safety of studies in $\mathrm{mBC}$. The results demonstrated that safety and efficacy outcomes with T-DM1 were, in general, more favorable than those with other treatments that are currently available/approved in this indication (pertuzumab plus trastuzumab plus capecitabine is not approved). This is particularly encouraging because T-DM1 is the first-in-class antibody-drug conjugate therapy approved in this indication.

In terms of efficacy, T-DM1 was associated with greater OS benefit than all approved comparators, both based on data at the end of the randomized phase of the EMILIA trial and after adjustment for pre-specified treatment crossover. T-DM1 was also associated with better PFS and ORR than other treatments.

The random-effects model results had wide CrIs, reflecting uncertainty around the comparisons. Fixed-effects results (Online Resource 9) which assume no between-study heterogeneity had similar hazard ratios but narrower CrIs.

In terms of safety outcomes, there is high uncertainty due to the small number of studies included, and the fact that not all endpoints were available for all comparator treatments. Discontinuation due to an $\mathrm{AE}$ or to an $\mathrm{AE}$ of at least grade 3 severity was less likely with T-DM1 than with other treatments, with the exception of neratinib, and T-DM1 generally had a more tolerable safety profile in terms of gastroenterological side effects than the other treatments, being substantially less likely to be associated with diarrhea. Similar point estimates for nausea and vomiting were seen with T-DM1 and trastuzumab plus capecitabine. Fatigue was generally less likely with T-DM1 than with comparators. Hematological effects were more difficult to quantify in this analysis. The likelihood of neutropenia with T-DM1 was slightly higher than with neratinib, and lower than with other approved treatments. When comparisons could be made, T-DM1 was more likely to be associated with anemia and thrombocytopenia than were other treatments. Finally, T-DM1 was among the treatments most likely to be associated with hepatic side effects.

Cardiac safety is an important consideration for patients treated with HER2 inhibitors, including T-DM1, who are at increased risk of developing left ventricular dysfunction. A decrease of left ventricular ejection fraction has been observed in patients treated with T-DM1. For example, in EMILIA, left ventricular dysfunction occurred in $1.8 \%$ of patients in the T-DM1-treated group and 3.3\% of patients in the lapitinib plus capecitabine group.

Generally, the risk of bias in different aspects of the RCTs included in the NMA was low. Response to treatment in GBG 26 (trastuzumab plus capecitabine versus capecitabine) [32] was at risk of being assessed by unblinded investigators due to a lack of central assessment; and CEREBEL (lapitinib plus capecitabine versus trastuzumab plus capecitabine) [17] was underpowered because it stopped before recruitment was complete, following interim analysis and a recommendation from its data monitoring committee. In several studies, risk of bias was unclear due to a lack of published information. This was typically related to details of randomization in open-label studies and to the methods of allocation concealment used, but little information about patients' baseline similarity was available in one study [39]. Differences in the follow-up times of the studies included in the NMA did not affect PFS and OS, which were analyzed using HRs that inherently assume proportional hazards. However, results for ORR and safety endpoints may have been affected. EMILIA was the largest study and had the longest follow-up, a fact that was reflected in the increased numbers of events observed in this study. Nonetheless, the efficacy and safety of T-DM1 were generally more favorable compared with the other treatments analyzed. It was not possible to analyze the rates of safety events to account for differences in the follow-up times, because not all studies reported the appropriate data to conduct such an analysis.

A key benefit of the current analysis is its reproducibility, which enables updates to be performed when additional evidence becomes available. A significant limitation of the NMA presented here is that a formal analysis of the likelihood of thrombocytopenia with T-DM1 versus other treatments could not be performed (i.e., effect size was not quantifiable due to thrombocytopenia being a rare event in most comparators). Thrombocytopenia was the most frequently reported grade 3 or above $\mathrm{AE}$ in patients treated with T-DM1 in the EMILIA trial, affecting 14\% of patients, compared with $<1 \%$ of patients in the lapatinib plus capecitabine group [13]. 


\section{Conclusion}

T-DM1 was associated with a greater OS, PFS, and ORR benefit than lapatinib plus capecitabine, trastuzumab plus capecitabine, capecitabine monotherapy, and neratinib monotherapy in patients with previously treated HER2-positive LABC or mBC. The improvements in OS with T-DM1 were seen in the analyses of both ITT (unadjusted) and after adjustment for treatment crossover. In the safety analyses, T-DM1 was associated with a greater benefit than the other treatments for the majority of endpoints with evaluable results with the exception of thrombocytopenia and hepatic AEs.

Acknowledgements The authors would like to acknowledge Christian Eichinger of PharmaGenesis London, London, UK, who provided medical writing support during the development of this manuscript which was funded by F. Hoffmann-La Roche AG . The authors would like to acknowledge Sandro Gsteiger from F. Hoffmann-La Roche AG for validating the analysis, and Christine Wohlfarth and Natsumi Irahara from F. Hoffmann-La Roche AG for their support with systematic review article screening process and heterogeneity assessment.

Funding This analysis was funded by F. Hoffmann-La Roche AG.

\section{Compliance with Ethical Standards}

Conflicts of interest NP is an employee of F. Hoffmann-La Roche AG and owns F. Hoffmann-La Roche AG stock. AR is an employee of F. Hoffmann-La Roche AG. VD Consultant/Advisory role: AbbVie, Astellas, AstraZeneca, Daiichi Sankyo, Eisai, Lilly, Merck Sharp \& Dohme, Nektar, Novartis, Odonate, Pfizer, Roche/Genentech, Seattle Genetics, Tesaro. IK Consultant/Advisory role: Bristol-Myers Squibb, Context Therapeutics, Daiichi Sankyo, MacroGenics, Merck, Novartis, Roche/Genentech, Seattle Genetics, Taiho Oncology. XP has nothing to declare. AU has received travel support from Roche.

Research involving human participants and/or animals This article does not contain any studies with human participants or animals performed by any of the authors.

Informed consent Not applicable.

Open Access This article is licensed under a Creative Commons Attribution 4.0 International License, which permits use, sharing, adaptation, distribution and reproduction in any medium or format, as long as you give appropriate credit to the original author(s) and the source, provide a link to the Creative Commons licence, and indicate if changes were made. The images or other third party material in this article are included in the article's Creative Commons licence, unless indicated otherwise in a credit line to the material. If material is not included in the article's Creative Commons licence and your intended use is not permitted by statutory regulation or exceeds the permitted use, you will need to obtain permission directly from the copyright holder. To view a copy of this licence, visit http://creativecommons.org/licenses/by/4.0/.

\section{References}

1. Moasser MM (2007) The oncogene HER2: its signaling and transforming functions and its role in human cancer pathogenesis. Oncogene 26(45):6469-6487. https://doi.org/10.1038/ sj.onc. 1210477

2. Wolff AC, Hammond ME, Hicks DG, Dowsett M, McShane LM, Allison KH, Allred DC, Bartlett JM, Bilous M, Fitzgibbons P, Hanna W, Jenkins RB, Mangu PB, Paik S, Perez EA, Press MF, Spears PA, Vance GH, Viale G, Hayes DF, American Society of Clinical Oncology, College of American Physicians (2013) Recommendations for human epidermal growth factor receptor 2 testing in breast cancer: American Society of Clinical Oncology/College of American Pathologists clinical practice guideline update. J Clin Oncol 31(31):3997-4013. https://doi.org/10.1200/ JCO.2013.50.9984

3. Slamon DJ, Clark GM, Wong SG, Levin WJ, Ullrich A, McGuire WL (1987) Human breast cancer: correlation of relapse and survival with amplification of the HER-2/neu oncogene. Science 235(4785):177-182

4. Roche_Pharma_AG (2018) Herceptin ${ }^{\circledR}$ summary of product characteristics. https://www.ema.europa.eu/en/documents/productinformation/herceptin-epar-product-information_en.pdf. Accessed 01 Apr 2019

5. Genentech_Inc (2010) HERCEPTIN prescribing information. https://www.accessdata.fda.gov/drugsatfda_docs/label /2010/103792s5250lbl.pdf. Accessed 01 Apr 2019

6. Moja L, Tagliabue L, Balduzzi S, Parmelli E, Pistotti V, Guarneri V, D'Amico R (2012) Trastuzumab containing regimens for early breast cancer. Cochrane Database Syst Rev. https://doi. org/10.1002/14651858.CD006243.pub2

7. National Comprehensive Cancer Network (2018) National Comprehensive Cancer Network Clinical Practice Guidelines in Oncology Breast Cancer Version 3. https://www.ncen.org/professionals/ physician_gls/pdf/breast.pdf. Accessed 20 Dec 2018

8. Lewis Phillips GD, Li G, Dugger DL, Crocker LM, Parsons KL, Mai E, Blattler WA, Lambert JM, Chari RV, Lutz RJ, Wong WL, Jacobson FS, Koeppen H, Schwall RH, Kenkare-Mitra SR, Spencer SD, Sliwkowski MX (2008) Targeting HER2-positive breast cancer with trastuzumab-DM1, an antibody-cytotoxic drug conjugate. Cancer Res 68(22):9280-9290. https://doi. org/10.1158/0008-5472.CAN-08-1776

9. Rinnerthaler G, Gampenrieder SP, Greil R (2019) HER2 directed antibody-drug-conjugates beyond T-DM1 in breast cancer. Int J Mol Sci 20(5):E1115. https://doi.org/10.3390/ijms20051115

10. Verma S, Miles D, Gianni L, Krop IE, Welslau M, Baselga J, Pegram M, Oh DY, Dieras V, Guardino E, Fang L, Lu MW, Olsen S, Blackwell K, EMILIA Study Group (2012) Trastuzumab emtansine for HER2-positive advanced breast cancer. N Engl J Med 367(19):1783-1791. https://doi.org/10.1056/NEJMoa1209 124

11. Food and Drug Administration (2013) Kadcyla. https://www. accessdata.fda.gov/drugsatfda_docs/label/2019/125427s105lbl. pdf. Accessed 06 Aug 2019

12. European Medicines Agency (2016) European public assessment report for Kadcyla. https://www.ema.europa.eu/en/documents/ overview/kadcyla-epar-summary-public_en.pdf. Accessed 20 Dec 2018

13. Dieras V, Miles D, Verma S, Pegram M, Welslau M, Baselga J, Krop IE, Blackwell K, Hoersch S, Xu J, Green M, Gianni L (2017) Trastuzumab emtansine versus capecitabine plus lapatinib in patients with previously treated HER2-positive advanced breast cancer (EMILIA): a descriptive analysis of final overall survival results from a randomised, open-label, phase 3 trial. Lancet Oncol 18(6):732-742. https://doi.org/10.1016/S1470-2045(17)30312-1 
14. von Minckwitz G, Huang CS, Mano MS, Loibl S, Mamounas EP, Untch M, Wolmark N, Rastogi P, Schneeweiss A, Redondo A, Fischer HH, Jacot W, Conlin AK, Arce-Salinas C, Wapnir IL, Jackisch C, DiGiovanna MP, Fasching PA, Crown JP, Wulfing P, Shao Z, Rota Caremoli E, Wu H, Lam LH, Tesarowski D, Smitt M, Douthwaite H, Singel SM, Geyer CE Jr (2019) Trastuzumab emtansine for residual invasive HER2-positive breast cancer. N Engl J Med 380(7):617-628. https://doi.org/10.1056/NEJMo a1814017

15. Food and Drug Administration (2019) FDA approves ado-trastuzumab emtansine for early breast cancer. https://www.fda.gov/ drugs/resources-information-approved-drugs/fda-approves-adotrastuzumab-emtansine-early-breast-cancer. Accessed 24 June 2019

16. NICE (2018) Managing advanced breast cancer. Hormone receptor-positive and HER2-positive disease. https://pathways.nice.org. uk/pathways/advanced-breast-cancer. Accessed 20 Dec 2018

17. Pivot X, Manikhas A, Zurawski B, Chmielowska E, Karaszewska B, Allerton R, Chan S, Fabi A, Bidoli P, Gori S, Ciruelos E, Dank M, Hornyak L, Margolin S, Nusch A, Parikh R, Nagi F, DeSilvio M, Santillana S, Swaby RF, Semiglazov V (2015) CEREBEL (EGF111438): a phase III, randomized, open-label study of lapatinib plus capecitabine versus trastuzumab plus capecitabine in patients with human epidermal growth factor receptor 2-positive metastatic breast cancer. J Clin Oncol 33(14):1564-1573. https:// doi.org/10.1200/JCO.2014.57.1794

18. Burstein HJ, Keshaviah A, Baron AD, Hart RD, Lambert-Falls R, Marcom PK, Gelman R, Winer EP (2007) Trastuzumab plus vinorelbine or taxane chemotherapy for HER2-overexpressing metastatic breast cancer: the trastuzumab and vinorelbine or taxane study. Cancer 110(5):965-972. https://doi.org/10.1002/ cncr.22885

19. Novartis_Europharm_Ltd (2019) Tyverb summary of product characteristics. https://www.ema.europa.eu/en/documents/produ ct-information/tyverb-epar-product-information_en.pdf. Accessed 01 Apr 2019

20. GlaxoSmithKline (2007) TYKERB prescribing information. https ://www.accessdata.fda.gov/drugsatfda_docs/label/2010/02205 9s007lbl.pdf. Accessed 01 Apr 2019

21. Puma_Biotechnology_Inc. (2018) Nerlynx ${ }^{\circledR}$ summary of product characteristics. https:/www.ema.europa.eu/en/documents/ product-information/nerlynx-epar-product-information_en.pdf. Accessed 01 Apr 2019

22. Puma_Biotechnology_Inc. (2017) NERLYNX prescribing information. https://www.accessdata.fda.gov/drugsatfda_docs/label /2017/208051s000lbl.pdf. Accessed 01 Apr 2019

23. Lumley $\mathrm{T}$ (2002) Network meta-analysis for indirect treatment comparisons. Stat Med 21(16):2313-2324. https://doi. org/10.1002/sim.1201

24. Roche_Pharma_AG (2018) Perjeta ${ }^{\circledR}$ summary of product characteristics. https://www.ema.europa.eu/en/documents/productinformation/perjeta-epar-product-information_en.pdf. Accessed 01 Apr 2019

25. Genentech_Inc. (2012) PERJETA prescribing information. https:// www.accessdata.fda.gov/drugsatfda_docs/label/2012/125409lbl. pdf. Accessed 01 Apr 2019

26. Urruticoechea A, Rizwanullah M, Im SA, Ruiz ACS, Lang I, Tomasello G, Douthwaite H, Badovinac Crnjevic T, Heeson S, Eng-Wong J, Munoz M (2017) Randomized phase III trial of trastuzumab plus capecitabine with or without pertuzumab in patients with human epidermal growth factor receptor 2-positive metastatic breast cancer who experienced disease progression during or after trastuzumab-based therapy. J Clin Oncol 35(26):3030-3038. https://doi.org/10.1200/JCO.2016.70.6267
27. Lewis MG, Sreekumaran N (2015) Review of applications of Bayesian meta-analysis in systematic reviews. Global Journal of Medicine and Public Health 4(1):1-9

28. van Valkenhoef G, Kuiper J (2016) Network meta-analysis using Bayesian methods. R package version 0.8-2. https://CRAN.Rproject.org/package=gemtc. Accessed 09 Apr 2019

29. R Core Team (2018) A language and environment for statistical computing. R Foundation for Statistical Computing, Vienna. https ://www.R-project.org/. Accessed 09 Apr 2019

30. Brooks SP, Gelman A (1998) General methods for monitoring convergence of iterative simulations. J Comput Graph Stat 7(4):434-455. https://doi.org/10.1080/10618600.1998.10474787

31. Turner RM, Jackson D, Wei Y, Thompson SG, Higgins JP (2015) Predictive distributions for between-study heterogeneity and simple methods for their application in Bayesian meta-analysis. Stat Med 34(6):984-998. https://doi.org/10.1002/sim.6381

32. von Minckwitz G, du Bois A, Schmidt M, Maass N, Cufer T, de Jongh FE, Maartense E, Zielinski C, Kaufmann M, Bauer W, Baumann KH, Clemens MR, Duerr R, Uleer C, Andersson M, Stein RC, Nekljudova V, Loibl S (2009) Trastuzumab beyond progression in human epidermal growth factor receptor 2-positive advanced breast cancer: a German Breast Group 26/Breast International Group 03-05 study. J Clin Oncol 27(12):1999-2006. https://doi.org/10.1200/JCO.2008.19.6618

33. NICE (2016) CDF Rapid Reconsideration of TA371: trastuzumab emtansine for the treatment of locally advanced or metastatic HER2-positive breast cancer after treatment with trastuzumab and a taxane (review of TA371). Committee Papers. https://www.nice. org.uk/guidance/ta458/documents/committee-papers-2. Accessed 19 Mar 2019

34. Robins JM, Tsiatis AA (1991) Correcting for non-compliance in randomized trials using rank preserving structural failure time models. Comm Stat Theor Meth 20:2609-2631

35. Latimer NR, Abrams KR (2014) NICE DSU technical support document 16: adjusting survival time estimates in the presence of treatment switching. http://nicedsu.org.uk/wp-content/uploa ds/2016/03/TSD16_Treatment_Switching.pdf. Accessed 14 May 2019

36. Cameron D, Casey M, Oliva C, Newstat B, Imwalle B, Geyer CE (2010) Lapatinib plus capecitabine in women with HER-2-positive advanced breast cancer: final survival analysis of a phase III randomized trial. Oncologist 15(9):924-934. https://doi.org/10.1634/ theoncologist.2009-0181

37. Salanti G, Ades AE, Ioannidis JP (2011) Graphical methods and numerical summaries for presenting results from multiple-treatment meta-analysis: an overview and tutorial. J Clin Epidemiol 64(2):163-171. https://doi.org/10.1016/j.jclinepi.2010.03.016

38. Cameron D, Casey M, Press M, Lindquist D, Pienkowski T, Romieu CG, Chan S, Jagiello-Gruszfeld A, Kaufman B, Crown J, Chan A, Campone M, Viens P, Davidson N, Gorbounova V, Raats JI, Skarlos D, Newstat B, Roychowdhury D, Paoletti P, Oliva C, Rubin S, Stein S, Geyer CE (2008) A phase III randomized comparison of lapatinib plus capecitabine versus capecitabine alone in women with advanced breast cancer that has progressed on trastuzumab: updated efficacy and biomarker analyses. Breast Cancer Res Treat 112(3):533-543. https://doi.org/10.1007/s1054 9-007-9885-0

39. Martin M, Bonneterre J, Geyer CE Jr, Ito Y, Ro J, Lang I, Kim SB, Germa C, Vermette J, Wang K, Wang K, Awada A (2013) A phase two randomised trial of neratinib monotherapy versus lapatinib plus capecitabine combination therapy in patients with HER2+ advanced breast cancer. Eur J Cancer 49(18):3763-3772. https:// doi.org/10.1016/j.ejca.2013.07.142

40. Takano T, Tsurutani J, Takahashi M, Yamanaka T, Sakai K, Ito Y, Fukuoka J, Kimura H, Kawabata H, Tamura K, Matsumoto K, Aogi K, Sato K, Nishio K, Nakagawa K, Saeki T (2017) A 
randomized phase II trial of trastuzumab + capecitabine versus lapatinib + capecitabine in patients with HER2-positive metastatic breast cancer previously treated with trastuzumab and taxanes: WJOG6110B/ELTOP. Cancer Res 77(4 Supplement):P4-21-14. https://doi.org/10.1158/1538-7445. SABCS16-P4-21-14

41. Krop IE, Kim SB, Gonzalez-Martin A, LoRusso PM, Ferrero JM, Smitt M, Yu R, Leung AC, Wildiers H, TH3RESA study collaborators (2014) Trastuzumab emtansine versus treatment of physician's choice for pretreated HER2-positive advanced breast cancer (TH3RESA): a randomised, open-label, phase 3 trial. Lancet Oncol 15(7):689-699. https://doi.org/10.1016/S1470 $-2045(14) 70178-0$
42. Turner RM, Davey J, Clarke MJ, Thompson SG, Higgins JP (2012) Predicting the extent of heterogeneity in meta-analysis, using empirical data from the Cochrane Database of Systematic Reviews. Int J Epidemiol 41(3):818-827. https://doi.org/10.1093/ ije/dys041

Publisher's Note Springer Nature remains neutral with regard to jurisdictional claims in published maps and institutional affiliations. 